\title{
SOLUTIONS OF THE DIVERGENCE AND KORN INEQUALITIES ON DOMAINS WITH AN EXTERNAL CUSP
}

\author{
Ricardo G. Durán and Fernando López García \\ Universidad de Buenos Aires, Departamento de Matemática \\ 1428 Buenos Aires, Argentina; rduran@dm.uba.ar \\ Universidad de Buenos Aires, Departamento de Matemática \\ 1428 Buenos Aires, Argentina; flopezg@dm.uba.ar
}

\begin{abstract}
This paper deals with solutions of the divergence for domains with external cusps. It is known that the classic results in standard Sobolev spaces, which are basic in the variational analysis of the Stokes equations, are not valid for this class of domains. For some bounded domains $\Omega \subset \mathbf{R}^{n}$ presenting power type cusps of integer dimension $m \leq n-2$, we prove the existence of solutions of the equation $\operatorname{div} \mathbf{u}=f$ in weighted Sobolev spaces, where the weights are powers of the distance to the cusp. The results obtained are optimal in the sense that the powers cannot be improved. As an application, we prove existence and uniqueness of solutions of the Stokes equations in appropriate spaces for cuspidal domains. Also, we obtain weighted Korn type inequalities for this class of domains.
\end{abstract}

\section{Introduction}

This paper deals with solutions of the divergence in domains with external cusps. Given a bounded domain $\Omega \subset \mathbf{R}^{n}$, it is known that, under appropriate assumptions on $\Omega$, there exists a continuous right inverse of the operator div : $W_{0}^{1, p}(\Omega)^{n} \rightarrow L_{0}^{p}(\Omega)$, $1<p<\infty$, where $L_{0}^{p}(\Omega)$ denotes the space of functions in $L^{p}(\Omega)$ with vanishing mean value in $\Omega$. In other words, given any $f \in L_{0}^{p}(\Omega)$, there exists a solution $\mathbf{u} \in W_{0}^{1, p}(\Omega)^{n}$ of

$$
\operatorname{div} \mathbf{u}=f
$$

satisfying

$$
\|\mathbf{u}\|_{W^{1, p}(\Omega)^{n}} \leq C\|f\|_{L^{p}(\Omega)},
$$

where the constant $C$ depends only on $\Omega$ and $p$.

This result has many applications, for example, in the particular case $p=2$, it is a basic tool for the variational analysis of the Stokes equations and it implies the Korn inequality in its more general form (see for example [BS, C]). Consequently, this problem has been widely studied and several methods to prove the existence of $\mathbf{u}$ satisfying (1.1) and (1.2), under different assumptions on the domain, have been developed (see for example [ADM, ASV, B, BS, DRS, GR, BA, L]). On the other hand, it is known that this result does not hold for domains with external cusps.

doi:10.5186/aasfm.2010.3527

2000 Mathematics Subject Classification: Primary 26D10, 35Q30; Secondary 76D03.

Key words: Divergence operator, weighted Sobolev spaces, Korn inequality.

Supported by ANPCyT under grant PICT 2006-01307, by Universidad de Buenos Aires under grant X070 and by CONICET under grant PIP 5478. The first author is a member of CONICET, Argentina. 
Several arguments have been given to show this fact [ADLg, D, GG], but the oldest counterexample goes back to Friedrichs, who showed that an inequality for analytic complex functions (which follows easily from the existence of $\mathbf{u}$ satisfying (1.1) and (1.2)) does not hold in a domain with a quadratic external cusp (see [F]). Therefore, it is an interesting question what kind of weaker results can be proved for these domains and whether these results can be applied to show the well posedness of the Stokes equations in appropriate spaces. Since the problem arises because of the bad behavior of the boundary, it seems natural to work with weighted Sobolev spaces where the weights are related to the distance to the boundary or to its singularities.

Recently, in [DLg], we have obtained results for planar simply connected Hölder$\alpha$ domains working with weights which are powers of the distance to the boundary of $\Omega$. The domains with external cusps that we are going to consider in this paper are a subclass of the Hölder- $\alpha$ domains. However, for this particular subclass, it is natural to look for stronger results where the distance to the boundary is replaced by the distance to the cusp, which can be a point or more generally a set of dimension $m \leq n-2$. To obtain this kind of results is the main goal of this paper.

As mentioned above, an important consequence of the existence of continuous right inverses of the divergence is the Korn inequality. We are going to show that the known arguments can be extended to some weighted cases allowing us to obtain new weighted Korn inequalities for domains with external cusps. Our results are optimal in the sense that the powers of the distance to the cusp involved in the estimates cannot be improved, this is proved in [ADLg]. As an application we prove the well posedness in appropriate spaces of the Stokes equations in domains with external cusps. In the particular two dimensional case similar results were proved in our previous paper [DLg] but a restriction in the power of the cusp was needed (this restriction is removed here).

The rest of the paper is organized as follows. Since the analysis of the Stokes equations is our main motivation, we start developing a generalized variational analysis of these equations, this is done in Section 2. Also in this section, we show by a simple example, that the existence of solution of the Stokes equations in the standard spaces is not true for cuspidal domains. In Section 3 we prove some auxiliary results concerning solutions of the divergence in weighted Sobolev spaces for domains which are star-shaped with respect to a ball. Section 4 contains our main results, namely, the existence of solutions of the divergence in appropriate spaces for cuspidal domains. Finally, Sections 5 and 6 deal with the applications to the Stokes equations and to the Korn inequalities respectively.

We will work with weighted $L^{p}$-norms. Given an almost everywhere positive function $\omega$ and a domain $\Omega \subset \mathbf{R}^{n}$ we denote with $L^{p}(\Omega, \omega)$ the Banach space with norm given by

$$
\|f\|_{L^{p}(\Omega, \omega)}^{p}=\int_{\Omega}|f(x)|^{p} \omega(x) d x .
$$

If $\omega$ is such that $L^{p}(\Omega, \omega) \subset L^{1}(\Omega), L_{0}^{p}(\Omega, \omega)$ denotes the subspace of $L^{p}(\Omega, \omega)$ of functions with vanishing mean value in $\Omega$.

\section{Generalized variational analysis of the Stokes equations}

The goal of this section is to explain the motivation of the main results of this paper, namely, the existence of right inverses of the divergence in weighted Sobolev 
spaces. First of all, we show by a simple example that the Stokes system of equations is not well posed in the usual Sobolev spaces for domains with external cusps. In view of this fact we introduce a generalization of the classic analysis for this kind of domains. We will use the usual notations for Sobolev spaces.

The Stokes equations are given by

$$
\begin{cases}-\Delta \mathbf{u}+\nabla p=\mathbf{g} & \text { in } \Omega, \\ \operatorname{div} \mathbf{u}=0 & \text { in } \Omega, \\ \mathbf{u}=0 & \text { on } \partial \Omega\end{cases}
$$

For a bounded domain $\Omega$ which is Lipschitz (or more generally a John domain [ADM]) it is known that, if $\mathbf{g} \in H^{-1}(\Omega)^{n}$, then there exists a unique solution

$$
(\mathbf{u}, p) \in H_{0}^{1}(\Omega)^{n} \times L_{0}^{2}(\Omega) .
$$

Moreover, the following a priori estimate holds

$$
\|\mathbf{u}\|_{H^{1}(\Omega)^{n}}+\|p\|_{L^{2}(\Omega)} \leq C\|\mathbf{g}\|_{H^{-1}(\Omega)^{n}}
$$

where the constant $C$ depends only on the domain $\Omega$.

Let us show that this result is not valid in general for domains with external cusps. Consider for example the domain

$$
\Omega=\left\{x=\left(x_{1}, x_{2}\right) \in \mathbf{R}^{2}: 0<x_{1}<1,\left|x_{2}\right|<x_{1}^{2}\right\} .
$$

It is known that there exists a function $p \in L_{0}^{1}(\Omega)$ such that $\nabla p \in H^{-1}(\Omega)^{2}$ but $p \notin L^{2}(\Omega)$. A simple example given by Acosta is $p\left(x_{1}, x_{2}\right)=\frac{1}{x_{1}^{2}}-3$. Indeed, by elementary integration one can easily check that $p \notin L^{2}(\Omega)$. On the other hand, to see that $\nabla p \in H^{-1}(\Omega)^{2}$, we only have to show that $\frac{\partial p}{\partial x_{1}} \in H^{-1}(\Omega)$, but this follows from $\frac{\partial p}{\partial x_{1}}=\frac{\partial}{\partial x_{2}}\left(-\frac{2 x_{2}}{x_{1}^{3}}\right)$ and $-\frac{2 x_{2}}{x_{1}^{3}} \in L^{2}(\Omega)$.

Consider now the Stokes problem (2.1) with

$$
\mathbf{g}(x)=\left(-\frac{2}{x_{1}^{3}}, 0\right)=\nabla p \in H^{-1}(\Omega)^{2} .
$$

Then,

$$
(\mathbf{u}, p)=\left(\mathbf{0}, \frac{1}{x_{1}^{2}}-3\right)
$$

is a solution, but $p \notin L^{2}(\Omega)$.

One could ask whether another solution in the space $H_{0}^{1}(\Omega)^{2} \times L_{0}^{2}(\Omega)$ exists. That this is not the case will follow from our general results which, for this particular domain, give existence and uniqueness (up to an additive constant in the pressure) in the space

$$
H_{0}^{1}(\Omega)^{2} \times L^{2}\left(\Omega,|x|^{2}\right) \supset H_{0}^{1}(\Omega)^{2} \times L^{2}(\Omega)
$$

and it is easy to see that our solution $(\mathbf{0}, p)$ belongs to this larger space.

Our general existence and uniqueness results for domains with cusps follow from the classic theory but replacing the usual Sobolev spaces by appropriate weighted spaces. The classic analysis of the Stokes equations is based on the abstract theory for saddle point problems given by Brezzi in [Br] (see also the books [BF, GR, BDF]). 
Indeed, for properly chosen $V$ and $Q$, the weak formulation of (2.1) can be written as

$$
\begin{cases}a(\mathbf{u}, \mathbf{v})+b(\mathbf{v}, p)=\int_{\Omega} \mathbf{g} \cdot \mathbf{v} & \forall \mathbf{v} \in V \\ b(\mathbf{u}, q)=0 & \forall q \in Q\end{cases}
$$

where

$$
a(\mathbf{u}, \mathbf{v})=\int_{\Omega} D \mathbf{u}: D \mathbf{v}
$$

and

$$
b(\mathbf{v}, p)=\int_{\Omega} p \operatorname{div} \mathbf{v}
$$

where, for $\mathbf{v} \in H^{1}(\Omega)^{n}, D \mathbf{v}$ is its differential matrix and, given two matrices $A=\left(a_{i j}\right)$ and $B=\left(b_{i j}\right)$ in $\mathbf{R}^{n \times n}, A: B=\sum_{i, j=1}^{n}=a_{i j} b_{i j}$.

The abstract theory gives existence and uniqueness for (2.3) when $a$ and $b$ are continuous bilinear forms, $a$ is coercive on the kernel of the operator $B: V \rightarrow Q^{\prime}$ associated with $b$, and $b$ satisfies the inf-sup condition

$$
\inf _{0 \neq q \in Q} \sup _{0 \neq \mathbf{v} \in V} \frac{b(\mathbf{v}, q)}{\|q\|_{Q}\|\mathbf{v}\|_{V}}>0 .
$$

In the case of the Stokes problem, if we choose the spaces $V=H_{0}^{1}(\Omega)^{n}$ and $Q=$ $L_{0}^{2}(\Omega)$, continuity of the bilinear forms and coercivity of $a$ follow immediately by Schwarz and Poincaré inequalities. Therefore, the problem reduces to prove the inf-sup condition for $b$ which reads

$$
\inf _{0 \neq q \in L_{0}^{2}(\Omega)} \sup _{0 \neq \mathbf{v} \in H_{0}^{1}(\Omega)^{n}} \frac{\int_{\Omega} q \operatorname{div} \mathbf{v}}{\|q\|_{L_{0}^{2}(\Omega)}\|\mathbf{v}\|_{H_{0}^{1}(\Omega)^{n}}}>0 .
$$

It is well known that this condition is equivalent to the existence of solutions of $\operatorname{div} \mathbf{u}=f$, for any $f \in L_{0}^{2}(\Omega)$, with $\mathbf{u} \in H_{0}^{1}(\Omega)^{n}$ satisfying $\|\mathbf{u}\|_{H^{1}(\Omega)^{n}} \leq C\|f\|_{L^{2}(\Omega)}$.

Observe that in the above example of cuspidal domain, the condition (2.4) does not hold, because it would imply existence of solution $(\mathbf{u}, p) \in H_{0}^{1}(\Omega)^{n} \times L_{0}^{2}(\Omega)$ for any $\mathbf{g} \in H^{-1}(\Omega)^{n}$ and we have shown that this is not possible. For domains such that (2.4) is not valid, the idea is to replace this condition by a weaker one. With this goal we will work with weighted norms.

We will use the following facts for $\omega \in L^{1}(\Omega)$ which are easy to see. First, $L^{2}\left(\Omega, \omega^{-1}\right) \subset L^{1}(\Omega)$ and therefore $L_{0}^{2}\left(\Omega, \omega^{-1}\right)$ is well defined, and second, the integral $\int_{\Omega} q \omega$ is well defined for $q \in L^{2}(\Omega, \omega)$ and therefore we can define the space

$$
L_{\omega, 0}^{2}(\Omega, \omega)=\left\{q \in L^{2}(\Omega, \omega): \int_{\Omega} q \omega=0\right\} .
$$

We have the following generalization of the classic result which will be useful for cuspidal domains.

Theorem 2.1. Let $\omega \in L^{1}(\Omega)$ be a positive weight. Assume that for any $f \in$ $L_{0}^{2}\left(\Omega, \omega^{-1}\right)$ there exists $\mathbf{u} \in H_{0}^{1}(\Omega)^{n}$ such that $\operatorname{div} \mathbf{u}=f$ and

$$
\|\mathbf{u}\|_{H^{1}(\Omega)^{n}} \leq C_{1}\|f\|_{L^{2}\left(\Omega, \omega^{-1}\right)}
$$

with a constant $C_{1}$ depending only on $\Omega$ and $\omega$. Then, for any $\mathbf{g} \in H^{-1}(\Omega)^{n}$, there exists a unique $(\mathbf{u}, p) \in H_{0}^{1}(\Omega)^{n} \times L_{\omega, 0}^{2}(\Omega, \omega)$ solution of the Stokes problem (2.1). 
Moreover,

$$
\|\mathbf{u}\|_{H^{1}(\Omega)^{n}}+\|p\|_{L^{2}(\Omega, \omega)} \leq C_{2}\|\mathbf{g}\|_{H^{-1}(\Omega)^{n}}
$$

where $C_{2}$ depends only on $C_{1}$ and $\Omega$.

Proof. We apply the general abstract theory for saddle point problems with appropriate spaces. For the pressure we introduce the space $Q=L_{\omega, 0}^{2}(\Omega, \omega)$ with the norm $\|q\|_{Q}=\|q\|_{L^{2}(\Omega, \omega)}$. Since we are modifying the pressure space, we have to enlarge the $H^{1}$-norm of the velocity space in order to preserve continuity of the bilinear form $b$. Then, we define

$$
V=\left\{\mathbf{v} \in H_{0}^{1}(\Omega)^{n}: \operatorname{div} \mathbf{v} \in L^{2}\left(\Omega, \omega^{-1}\right)\right\}
$$

with the norm given by

$$
\|\mathbf{v}\|_{V}^{2}=\|\mathbf{v}\|_{H^{1}(\Omega)^{n}}^{2}+\|\operatorname{div} \mathbf{v}\|_{L^{2}\left(\Omega, \omega^{-1}\right)}^{2} .
$$

Since $\|\mathbf{v}\|_{H^{1}(\Omega)^{n}} \leq\|\mathbf{v}\|_{V}$ the continuity of $a$ in $V \times V$ follows immediately by Schwarz inequality. Also, from the definitions of the spaces it is easy to see that $b$ is continuous on $V \times Q$.

On the other hand, coercivity of $a$, in the norm of $V$, on the kernel of the operator $B$ follows from Poincaré inequality because this kernel consists of divergence free vector fields. Therefore, to apply the general theory it only rests to prove the inf-sup condition

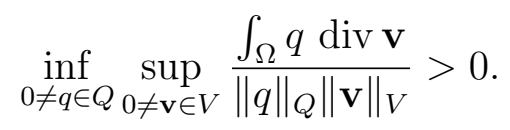

But this follows in a standard way. Indeed, given $q \in Q$ it follows from our hypothesis that there exists $\mathbf{u} \in H_{0}^{1}(\Omega)^{n}$ such that $\operatorname{div} \mathbf{u}=q \omega$ and

$$
\|\mathbf{u}\|_{H^{1}(\Omega)^{n}} \leq C_{1}\|q \omega\|_{L^{2}\left(\Omega, \omega^{-1}\right)}=C_{1}\|q\|_{Q} .
$$

Moreover, since $\|\operatorname{div} \mathbf{u}\|_{L^{2}\left(\Omega, \omega^{-1}\right)}=\|q\|_{Q}$ we have

$$
\|\mathbf{u}\|_{V} \leq C\|q\|_{Q}
$$

with $C$ depending only on $C_{1}$. Then,

$$
\|q\|_{Q}=\frac{\int_{\Omega} q q \omega}{\|q\|_{Q}} \leq C \frac{\int_{\Omega} q \operatorname{div} \mathbf{u}}{\|\mathbf{u}\|_{V}}
$$

and therefore (2.5) holds.

As an example let us mention that the hypothesis of the theorem holds for the case of the cuspidal domain introduced in (2.2) with $\omega(x)=|x|^{2}$. This result is a particular case of the general results that we are going to prove in Section 4 . Consequently, there is a unique weak solution $(\mathbf{u}, p) \in H_{0}^{1}(\Omega)^{2} \times L_{0}^{2}\left(\Omega,|x|^{2}\right)$ of the Stokes equations (2.1) in this domain.

\section{Solutions of the divergence in star-shaped domains}

We will work with weighted Sobolev spaces. Given weights $\omega_{1}, \omega_{2}: \mathbf{R}^{n} \rightarrow[0, \infty]$, for any domain $U \subset \mathbf{R}^{n}$ and $1<p<\infty$, we define

$$
W^{1, p}\left(U, \omega_{1}, \omega_{2}\right)=\left\{f \in L^{p}\left(U, \omega_{1}\right): \frac{\partial f}{\partial x_{i}} \in L^{p}\left(U, \omega_{2}\right), 1 \leq i \leq n\right\}
$$


with the norm given by

$$
\|f\|_{W^{1, p}\left(U, \omega_{1}, \omega_{2}\right)}^{p}=\int_{U}|f(x)|^{p} \omega_{1}(x) d x+\sum_{i=1}^{n} \int_{U}\left|\frac{\partial f(x)}{\partial x_{i}}\right|^{p} \omega_{2}(x) d x .
$$

To simplify notation we will write $W^{1, p}(U, \omega)$ instead of $W^{1, p}(U, \omega, \omega)$.

To prove our main results concerning solutions of the divergence in cuspidal domains, we will make use of the existence of solutions in weighted Sobolev spaces for good domains. We will work with weights in the Muckenhoupt class $A_{p}$ (see for example [Du, S2]). Recall that, for $1<p<\infty$, a non-negative weight defined in $\mathbf{R}^{n}$ is in $A_{p}$ if

$$
\sup _{B \subset \mathbf{R}^{n}}\left(\frac{1}{|B|} \int_{B} \omega\right)\left(\frac{1}{|B|} \int_{B} \omega^{-1 /(p-1)}\right)^{p-1}<\infty,
$$

where the supremum is taken over all the balls $B \subset \mathbf{R}^{n}$ and $|B|$ denotes the Lebesgue measure of $B$. It is known that, if $\omega \in A_{p}$, the spaces $W^{1, p}(U, \omega)$ and $L^{p}(U, \omega)$ are Banach spaces (see $[\mathrm{GU}]$ ).

Remark 3.1. If $U$ is a bounded domain and $\omega \in A_{p}$ then, $L^{p}(U, \omega) \subset L^{1}(U)$. Indeed, let $B$ a ball containing $U$. We have,

$$
\begin{aligned}
\int_{U}|f| & =\int_{U}|f| \omega^{1 / p} \omega^{-1 / p} \leq\left(\int_{U}|f|^{p} \omega\right)^{1 / p}\left(\int_{U} \omega^{-p^{\prime} / p}\right)^{1 / p^{\prime}} \\
& \leq|B|^{(p-1) / p}\|f\|_{L^{p}(U, \omega)}\left(\frac{1}{|B|} \int_{B} \omega^{-1 /(p-1)}\right)^{(p-1) / p} .
\end{aligned}
$$

In view of this remark the space $L_{0}^{p}(U, \omega)$ is well defined. We will work also with the space $W_{0}^{1, p}(U, \omega)$ defined as the closure of $C_{0}^{\infty}(U)$ in $W^{1, p}(U, \omega)$. Now we give the auxiliary result that we need. We state it as a theorem since it can be of interest in itself. We outline a proof based on Bogovskii's formula for solutions of the divergence [B, DM, G]. An alternative proof of this result was given in [DRS].

Theorem 3.1. Let $\omega \in A_{p}, 1<p<\infty$, and $U \subset \mathbf{R}^{n}$ be a bounded domain which is star-shaped with respect to a ball $B \subset U$. Given $f \in L_{0}^{p}(U, \omega)$, there exists $\mathbf{u} \in W_{0}^{1, p}(U, \omega)^{n}$ satisfying

$$
\operatorname{div} \mathbf{u}=f
$$

and

$$
\|\mathbf{u}\|_{W^{1, p}(U, \omega)^{n}} \leq C\|f\|_{L^{p}(U, \omega)},
$$

with a constant $C$ depending only on $\omega, U$ and $p$.

Proof. Using general results for singular integral operators we are going to show that the explicit solution of $\operatorname{div} \mathbf{u}=f$ introduced by Bogovskii in [B] (see also $[\mathrm{DM}, \mathrm{G}]$ ) satisfies the desired property. In what follows we consider $f$ defined in $\mathbf{R}^{n}$ extending it by zero to the complement of $U$.

Bogovskii's solution can be written as

$$
\mathbf{u}(x)=\int_{U} \mathbf{G}(x, y) f(y) d y
$$


with $\mathbf{G}(x, y)=\left(G_{1}, \ldots, G_{n}\right)$ given by

$$
\mathbf{G}(x, y)=\psi(y) \int_{0}^{1} \frac{(x-y)}{s^{n+1}} \phi\left(y+\frac{x-y}{s}\right) d s,
$$

where $\varphi \in C_{0}^{\infty}(B)$ is such that $\int_{B} \varphi=1$ and $\psi \in C_{0}^{\infty}\left(\mathbf{R}^{n}\right)$ is a regularized characteristic of $U$, i.e., $\psi(y)=1$ for any $y \in U$ and $\psi$ is supported in a neighborhood of $U$. In what follows the letter $C$ denotes a generic constant which may depend on $n, p$, $\varphi, \omega$, and the diameter of $U$, that we will call $d$, but is independent of $f$ and $\mathbf{u}$.

Let us first see that $\mathbf{u} \in L^{p}(U, \omega)^{n}$. It is known that (see [DM, G])

$$
|\mathbf{G}(x, y)| \leq \frac{C}{|x-y|^{n-1}} .
$$

Using (3.2) we have, for $x \in U$,

$$
\begin{aligned}
|\mathbf{u}(x)| & \leq C \int_{\mathrm{U}} \frac{1}{|x-y|^{n-1}}|f(y)| d y \leq C \int_{B(x, d)} \frac{1}{|x-y|^{n-1}}|f(y)| d y \\
& \leq C \sum_{k=0}^{\infty} \int_{\frac{d}{2^{k+1}}<|y-x|<\frac{d}{2^{k}}} \frac{1}{|x-y|^{n-1}}|f(y)| d y \\
& \leq C \sum_{k=0}^{\infty} \int_{\frac{d}{2^{k+1}}<|y-x|<\frac{d}{2^{k}}}\left(\frac{2^{k+1}}{d}\right)^{n-1}|f(y)| d y \\
& \leq C \sum_{k=0}^{\infty} 2^{-k} \frac{1}{\left|B\left(x, \frac{d}{2^{k}}\right)\right|} \int_{B\left(x, \frac{d}{2^{k}}\right)}|f(y)| d y \leq C M f(x),
\end{aligned}
$$

where $M f$ denotes the Hardy-Littlewood maximal function of $f$. Since $\omega \in A_{p}$, the maximal operator is bounded in $L^{p}\left(\mathbf{R}^{n}, \omega\right)$ (see for example [Du, S2]), and therefore

$$
\|\mathbf{u}\|_{L^{p}(U, \omega)^{n}} \leq C\|f\|_{L^{p}(U, \omega)} .
$$

Now, to see that the first derivatives of the components $u_{j}$ of $\mathbf{u}$ are also in $L^{p}(U, \omega)$ we use that this derivatives can be written in the following way (see [DM, G]),

$$
\frac{\partial u_{j}}{\partial x_{i}}=\varphi_{i j} f+T_{i j} f
$$

where $\varphi_{i j}$ is a function bounded by a constant depending only on $\varphi$ and

$$
T_{i j} f(x)=\lim _{\varepsilon \rightarrow 0} \int_{|y-x|>\varepsilon} \frac{\partial G_{j}}{\partial x_{i}}(x, y) f(y) d y .
$$

Therefore, to prove (3.1) it only remains to prove that the operators $T_{i j}$ are bounded in $L^{p}\left(\mathbf{R}^{n}, \omega\right)$.

It was shown in [DM, G] that $T_{i j}$ is continuous in $L^{p}\left(\mathbf{R}^{n}\right)$ by using the CalderónZygmund singular integral operator theory developed in [CZ]. We were not able to find in the literature that a general operator of the form considered in [CZ] is continuous in $L^{p}\left(\mathbf{R}^{n}, \omega\right)$ for $\omega \in A_{p}$. However, such a continuity result is known to hold for an operator of the form

$$
T f(x)=\lim _{\varepsilon \rightarrow 0} \int_{|y-x|>\varepsilon} K(x, y) f(y) d y
$$


which is bounded in $L^{2}\left(\mathbf{R}^{n}\right)$ and with a kernel satisfying

$$
|K(x, y)| \leq \frac{C}{|x-y|^{n}},
$$

and the so called Hörmander conditions, namely,

$$
\left|K(x, y)-K\left(x^{\prime}, y\right)\right| \leq C \frac{\left|x-x^{\prime}\right|}{|x-y|^{n+1}} \quad \text { if }|x-y| \geq 2\left|x-x^{\prime}\right|
$$

and

$$
\left|K(x, y)-K\left(x, y^{\prime}\right)\right| \leq C \frac{\left|y-y^{\prime}\right|}{|x-y|^{n+1}} \quad \text { if }|x-y| \geq 2\left|y-y^{\prime}\right|,
$$

see [S2, page 221]. For $T_{i j}$ we have

$$
K(x, y)=\frac{\partial G_{j}}{\partial x_{i}}(x, y)=\psi(y) \int_{0}^{1} \frac{\delta_{i j}}{s^{n+1}} \varphi\left(y+\frac{x-y}{s}\right)+\frac{x_{j}-y_{j}}{s^{n+2}} \frac{\partial \varphi}{\partial x_{i}}\left(y+\frac{x-y}{s}\right) d s,
$$

where $\delta_{i j}$ denotes the Kronecker symbol. This kernel satisfies (3.4) ([DM, G]) and also the Hörmander conditions (this was proved in $[\mathrm{N}]$ ). In conclusion, we obtain that, for any $i, j$,

$$
\left\|\frac{\partial u_{j}}{\partial x_{i}}\right\|_{L^{p}(U, \omega)} \leq C\|f\|_{L^{p}(U, \omega)}
$$

which together with (3.3) gives (3.1).

To end the proof we have to show that $\mathbf{u}$ vanishes at the boundary, i.e., $\mathbf{u} \in$ $W_{0}^{1, p}(U, \omega)^{n}$. For an arbitrary weight this is not obvious from the definition of $\mathbf{u}$. However, once that we know the estimate (3.1) we can prove it by density. We omit details because they are standard.

Remark 3.2. If the weight $\omega$ in the previous Theorem is a power of the distance to the origin (which is one of the case of interest in our applications to Stokes) it is not necessary to use the Hörmander conditions. Indeed, in this case (3.1) can be proved using the results in ([S1]).

\section{Solutions of the divergence in cuspidal domains}

In this section we prove the existence of solutions of the divergence in weighted Sobolev spaces for domains with an external cusp. We consider the following class of domains. Given integer numbers $k \geq 1$ and $m \geq 0$ we define

$$
\Omega=\left\{(x, y, z) \in I \times \mathbf{R}^{k} \times I^{m}:|y|<x^{\gamma}\right\} \subset \mathbf{R}^{n},
$$

where $n=m+k+1, I$ is the interval $(0,1)$ and $\gamma \geq 1$. For $\gamma=1, \Omega$ is a convex domain while, for $\gamma>1, \Omega$ has an external cusp. The set of singularities of the boundary, which has dimension $m$, will be called $M$. Namely,

$$
M=\{\mathbf{0}\} \times[0,1]^{m} \subset \mathbf{R}^{k+1} \times \mathbf{R}^{m} .
$$

We will work with weighted Sobolev spaces where the weights are powers of the distance to $M$ that will be called $d_{M}$. Precisely, we will use the spaces $L^{p}\left(\Omega, d_{M}^{p \beta}\right)$ and $W^{1, p}\left(\Omega, d_{M}^{p \beta_{1}}, d_{M}^{p \beta_{2}}\right)$ where $\beta, \beta_{1}$ and $\beta_{2}$ are real numbers. For $\beta_{1}=\beta_{2}=\beta$ we will write $W^{1, p}\left(\Omega, d_{M}^{p \beta}\right)$ instead of $W^{1, p}\left(\Omega, d_{M}^{p \beta}, d_{M}^{p \beta}\right)$. It is well known that these spaces are Banach spaces (see $[\mathrm{Ku}]$, Theorem 3.6. for details). 
We consider $d_{M}$ defined everywhere in $\mathbf{R}^{n}$ and we are going to use the following result that we state as a lemma for the sake of clarity.

Lemma 4.1. If $-(n-m)<\mu<(n-m)(p-1)$, then $d_{M}^{\mu} \in A_{p}$.

Proof. It follows from the more general result proved in Lemma 3.3 of [DLg]. Indeed, calling $d_{F}$ the distance to a compact set $F \subset \mathbf{R}^{n}$, it was proved in that paper that $d_{F}^{\mu} \in A_{p}$ whenever the $m$-dimensional Hausdorff measure of $B(x, r) \cap F$ is equivalent to $r^{m}$, for all $x \in F$ and $r<\operatorname{diam}(F)$.

In what follows we will use several times that, for $(x, y, z) \in \Omega, d_{M}(x, y, z) \simeq x$, where the symbol $\simeq$ denotes equivalence up to multiplicative constants. Indeed, it is easy to see that $x \leq d_{M}(x, y, z)=|(x, y)| \leq(\sqrt{2}) x$. In the proof of the main result of this section we will use the Hardy type inequality given in the next lemma.

Lemma 4.2. Let $\Omega$ be the domain defined in (4.1) and $1<p<\infty$. Given $\kappa \in \mathbf{R}$, if $v \in W_{0}^{1, p}\left(\Omega, d_{M}^{p \kappa}\right)$ then, $v / x \in L^{p}\left(\Omega, d_{M}^{p \kappa}\right)$ and there exists constant $C$, depending only on $p$, such that

$$
\left\|\frac{v}{x}\right\|_{L^{p}\left(\Omega, d_{M}^{p \kappa}\right)} \leq C\left\|\frac{\partial v}{\partial y_{1}}\right\|_{L^{p}\left(\Omega, d_{M}^{p \kappa}\right)} .
$$

Consequently, $W_{0}^{1, p}\left(\Omega, d_{M}^{p \kappa}\right)$ is continuously imbedded in $W_{0}^{1, p}\left(\Omega, d_{M}^{p(\kappa-1)}, d_{M}^{p \kappa}\right)$.

Proof. By density it is enough to prove (4.3) for $v \in C_{0}^{\infty}(\Omega)$. Writing $x^{p \kappa-p}=$ $\frac{\partial\left(x^{p \kappa-p} y_{1}\right)}{\partial y_{1}}$ and integrating by parts we have

$$
\begin{aligned}
\int_{\Omega}|v(x, y, z)|^{p} x^{p \kappa-p} d x d y d z & =\int_{\Omega}|v(x, y, z)|^{p} \frac{\partial\left(x^{p \kappa-p} y_{1}\right)}{\partial y_{1}} d x d y d z \\
& =-\int_{\Omega} \frac{\partial\left(|v(x, y, z)|^{p}\right)}{\partial y_{1}} x^{p \kappa-p} y_{1} d x d y d z .
\end{aligned}
$$

Then, since $\left|y_{1}\right| \leq x$, we have

$$
\int_{\Omega}|v(x, y, z)|^{p} x^{p \kappa-p} d x d y d z \leq p \int_{\Omega}|v(x, y, z)|^{p-1}\left|\frac{\partial v(x, y, z)}{\partial y_{1}}\right| x^{p \kappa-p+1} d x d y d z
$$

and so, writing now $x^{p \kappa-p+1}=x^{\frac{p \kappa-p}{p^{\prime}}} x^{\kappa}$ and applying the Hölder inequality, we obtain

$$
\begin{aligned}
& \int_{\Omega}|v(x, y, z)|^{p} x^{p \kappa-p} d x d y d z \\
& \leq p\left(\int_{\Omega}|v(x, y, z)|^{p} x^{p \kappa-p} d x d y d z\right)^{(p-1) / p}\left(\int_{\Omega}\left|\frac{\partial v(x, y, z)}{\partial y_{1}}\right|^{p} x^{p \kappa} d x d y d z\right)^{1 / p}
\end{aligned}
$$

and therefore,

$$
\left(\int_{\Omega}|v(x, y, z)|^{p} x^{p \kappa-p} d x d y d z\right)^{1 / p} \leq p\left(\int_{\Omega}\left|\frac{\partial v(x, y, z)}{\partial y_{1}}\right|^{p} x^{p \kappa} d x d y d z\right)^{1 / p} .
$$

To conclude the proof we use that $d_{M}(x, y, z) \simeq x$ and then, that $W_{0}^{1, p}\left(\Omega, d_{M}^{p \kappa}\right)$ is continuously imbedded in $W_{0}^{1, p}\left(\Omega, d_{M}^{p(\kappa-1)}, d_{M}^{p \kappa}\right)$ follows from (4.3).

We can now prove the main result of this section. 
Theorem 4.1. Let $\Omega$ be the domain defined in (4.1) for a fixed $\gamma>1, M$ defined as in (4.2), and $1<p<\infty$. If $\beta \in\left(\frac{-\gamma(n-m)}{p}-\frac{\gamma-1}{p^{\prime}}, \frac{\gamma(n-m)}{p^{\prime}}-\frac{\gamma-1}{p^{\prime}}\right)$ and $\eta \in \mathbf{R}$ is such that $\eta \geq \beta+\gamma-1$ then, given $f \in L_{0}^{p}\left(\Omega, d_{M}^{p \beta}\right)$, there exists $\mathbf{u} \in W_{0}^{1, p}\left(\Omega, d_{M}^{p(\eta-1)}, d_{M}^{p \eta}\right)^{n}$ satisfying

$$
\operatorname{div} \mathbf{u}=f
$$

and

$$
\|\mathbf{u}\|_{W^{1, p}\left(\Omega, d_{M}^{p(\eta-1)}, d_{M}^{p \eta}\right)^{n}} \leq C\|f\|_{L^{p}\left(\Omega, d_{M}^{p \beta}\right)}
$$

with a constant $C$ depending only on $\gamma, \beta, \eta, p$ and $n$.

Proof. It is enough to prove the result for the case $\eta=\beta+\gamma-1$. Therefore we are going to consider this case. Define

$$
\widehat{\Omega}=\left\{(\hat{x}, \hat{y}, \hat{z}) \in I \times \mathbf{R}^{k} \times I^{m}:|\hat{y}|<\hat{x}\right\} \subset \mathbf{R}^{n}
$$

and let $F: \widehat{\Omega} \rightarrow \Omega$ be the one-to-one application given by

$$
F(\hat{x}, \hat{y}, \hat{z})=\left(\hat{x}^{\alpha}, \hat{y}, \hat{z}\right)=(x, y, z)
$$

where $\alpha=1 / \gamma$.

By this change of variables we associate functions defined in $\Omega$ with functions defined in $\widehat{\Omega}$ in the following way,

$$
h(x, y, z)=\hat{h}(\hat{x}, \hat{y}, \hat{z}) .
$$

Now, for $f \in L_{0}^{p}\left(\Omega, d_{M}^{p \beta}\right)$, we define $\hat{g}: \widehat{\Omega} \rightarrow \Omega$ by

$$
\hat{g}(\hat{x}, \hat{y}, \hat{z}):=\alpha \hat{x}^{\alpha-1} \hat{f}(\hat{x}, \hat{y}, \hat{z}) .
$$

We want to apply Theorem 3.1 for $\hat{g}$ on the convex domain $\widehat{\Omega}$ and then obtain the desired solution of (4.4) by using the so called Piola transform for vector fields.

In the rest of the proof we will use several times that, for $(x, y, z) \in \Omega, d_{M}(x, y, z)$ $\simeq x$, det $D F(\hat{x}, \hat{y}, \hat{z})=\alpha \hat{x}^{\alpha-1}$ and $\operatorname{det} D F^{-1}(x, y, z)=\gamma x^{\gamma-1}$. First let us see that, for $\hat{\beta}=\alpha\left(\beta+(\gamma-1) / p^{\prime}\right)$, we have

$$
\hat{g} \in L_{0}^{p}\left(\widehat{\Omega}, d_{M}^{p \hat{\beta}}\right) \quad \text { and } \quad\|\hat{g}\|_{L^{p}\left(\widehat{\Omega}, d_{M}^{p \hat{\beta}}\right)} \simeq\|f\|_{L^{p}\left(\Omega, d_{M}^{p \beta}\right)} .
$$

Indeed, we have

$$
\begin{aligned}
\|\hat{g}\|_{L^{p}\left(\widehat{\Omega}, d_{M}^{p \hat{\beta}}\right)}^{p} & \simeq \int_{\widehat{\Omega}}|\hat{g}|^{p} \hat{x}^{p \hat{\beta}}=\alpha^{p} \int_{\widehat{\Omega}}|\hat{f}|^{p} \hat{x}^{p(\alpha-1)} \hat{x}^{\alpha p\left(\beta+(\gamma-1) / p^{\prime}\right)} \\
& =\alpha^{p} \int_{\Omega}|f|^{p} x^{p \beta+1-\gamma} \gamma x^{\gamma-1} \simeq\|f\|_{L^{p}\left(\Omega, d_{M}^{p \beta}\right)}^{p}
\end{aligned}
$$

and

$$
\int_{\widehat{\Omega}} \hat{g}=\alpha \int_{\widehat{\Omega}} \hat{f} \hat{x}^{\alpha-1}=\alpha \int_{\Omega} f x^{1-\gamma} \gamma x^{\gamma-1}=\int_{\Omega} f=0 .
$$

Thus, (4.7) holds.

Observe that, from Lemma 4.1 and our hypothesis on $\beta$, we have $d_{M}^{p \hat{\beta}} \in A_{p}$. In particular, it follows from Remark 3.1 that $\hat{g} \in L^{1}(\widehat{\Omega})$ and therefore the mean value of $f$ in $\Omega$ is well defined. 
Now, from Theorem 3.1 we know that there exists $\hat{\mathbf{v}} \in W_{0}^{1, p}\left(\widehat{\Omega}, d_{M}^{p \hat{\beta}}\right)^{n}$ such that

$$
\operatorname{div} \hat{\mathbf{v}}=\hat{g}
$$

and

$$
\|\hat{\mathbf{v}}\|_{W^{1, p}\left(\widehat{\Omega}, d_{M}^{p \hat{\beta}}\right)^{n}} \leq C\|\hat{g}\|_{L^{p}\left(\widehat{\Omega}, d_{M}^{p \hat{\beta}}\right.} .
$$

Now, we define $\mathbf{u}$ as the Piola transform of $\hat{\mathbf{v}}$, namely,

$$
\mathbf{u}(x, y, z)=\frac{1}{\operatorname{det} D F} D F(\hat{x}, \hat{y}, \hat{z}) \hat{\mathbf{v}}(\hat{x}, \hat{y}, \hat{z})
$$

or equivalently, if $\hat{\mathbf{v}}=\left(\hat{v}_{1}, \ldots, \hat{v}_{n}\right)$,

$$
\mathbf{u}(x, y, z)=\gamma x^{\gamma-1}\left(\alpha x^{1-\gamma} \hat{v}_{1}\left(x^{\gamma}, y, z\right), \hat{v}_{2}\left(x^{\gamma}, y, z\right), \ldots, \hat{v}_{n}\left(x^{\gamma}, y, z\right)\right) .
$$

Then, using (4.8), it is easy to see that

$$
\operatorname{div} \mathbf{u}=f .
$$

To prove (4.5) we first show that

$$
\|\mathbf{u}\|_{W^{1, p}\left(\Omega, d_{M}^{p \eta}\right)^{n}} \leq C\|f\|_{L^{p}\left(\Omega, d_{M}^{p \beta}\right)} .
$$

In view of the equivalence of norms given in (4.7) and the estimate (4.9), to prove (4.10) it is enough to see that

$$
\|\mathbf{u}\|_{W^{1, p}\left(\Omega, d_{M}^{p \eta}\right)^{n}} \leq C\|\hat{\mathbf{v}}\|_{W^{1, p}\left(\widehat{\Omega}, d_{M}^{p \hat{\beta}}\right)^{n}} .
$$

But, we have

$$
\left\|u_{1}\right\|_{L^{p}\left(\Omega, d_{M}^{p \eta}\right)}^{p} \simeq \int_{\Omega}\left|u_{1}\right|^{p} x^{p \eta}=\alpha \int_{\widehat{\Omega}}\left|\hat{v}_{1}\right|^{p} \hat{x}^{\alpha p \eta} \hat{x}^{\alpha-1} \simeq\left\|\hat{v}_{1}\right\|_{L^{p}\left(\Omega, d_{M}^{p \hat{\beta}}\right)}^{p},
$$

where in the last step we have used $\alpha p \eta+\alpha-1=p \hat{\beta}$. In an analogous way we can show that, for $j=2, \ldots, n$,

$$
\left\|u_{j}\right\|_{L^{p}\left(\Omega, d_{M}^{p \eta}\right)} \leq C\left\|\hat{v}_{j}\right\|_{L^{p}\left(\Omega, d_{M}^{p \hat{\beta}}\right)}
$$

Then, it only remains to bound the derivatives of the components of $\mathbf{u}$. That

$$
\left\|\frac{\partial u_{1}}{\partial y_{1}}\right\|_{L^{p}\left(\Omega, d_{M}^{p \eta}\right)}^{p} \simeq\left\|\frac{\partial \hat{v}_{1}}{\partial \hat{y}_{1}}\right\|_{L^{p}\left(\widehat{\Omega}, d_{M}^{p \hat{\beta}}\right)}^{p}
$$

follows exactly as (4.12). Let us now estimate $\frac{\partial u_{2}}{\partial x}$. Using

$$
\left|\frac{\partial u_{2}}{\partial x}\right|=\gamma^{2}\left|\frac{\gamma-1}{\gamma} \frac{\hat{v}_{2}\left(x^{\gamma}, y, z\right)}{x^{\gamma}}+\frac{\partial \hat{v}_{2}\left(x^{\gamma}, y, z\right)}{\partial \hat{x}}\right| x^{2(\gamma-1)}
$$

and Lemma 4.2 for $\widehat{\Omega}$ we have

$$
\begin{aligned}
\left\|\frac{\partial u_{2}}{\partial x}\right\|_{L^{p}\left(\Omega, d_{M}^{p \eta}\right)}^{p} & \simeq \int_{\Omega}\left|\frac{\partial u_{2}}{\partial x}\right|^{p} x^{p \eta} \leq C \int_{\widehat{\Omega}}\left(\left|\frac{\hat{v}_{2}}{\hat{x}}\right|^{p}+\left|\frac{\partial \hat{v}_{2}}{\partial \hat{x}}\right|^{p}\right) \hat{x}^{2 p(1-\alpha)} \hat{x}^{\alpha p \eta+\alpha-1} \\
& \leq C \int_{\widehat{\Omega}}\left(\left|\frac{\hat{v}_{2}}{\hat{x}}\right|^{p}+\left|\frac{\partial \hat{v}_{2}}{\partial \hat{x}}\right|^{p}\right) \hat{x}^{p \hat{\beta}} \leq C\left(\left\|\frac{\partial \hat{v}_{2}}{\partial \hat{y}_{1}}\right\|_{L^{p}\left(\widehat{\Omega}, d_{M}^{p \hat{\beta}}\right)}^{p}+\left\|\frac{\partial \hat{v}_{2}}{\partial \hat{x}}\right\|_{L^{p}\left(\hat{\Omega}, d_{M}^{p \hat{\beta}}\right)}^{p}\right),
\end{aligned}
$$

where we have used again $\alpha p \eta+\alpha-1=p \hat{\beta}$ and that $2 p(1-\alpha)>0$. All the other derivatives of the components of $\mathbf{u}$ can be bounded in an analogous way and therefore (4.11) holds. 
Now, since

$$
\left.\mathbf{u}\right|_{\partial \Omega}=\left.\frac{1}{\operatorname{det} D F} D F \hat{\mathbf{v}}\right|_{\partial \widehat{\Omega}}
$$

it is easy to check that $\mathbf{u}$ belongs to the closure of $C_{0}^{\infty}(\Omega)^{n}$, i.e., $\mathbf{u} \in W_{0}^{1, p}\left(\Omega, d_{M}^{p \eta}\right)^{n}$ and by Lemma $4.2 \mathbf{u} \in W_{0}^{1, p}\left(\Omega, d_{M}^{p(\eta-1)}, d_{M}^{p \eta}\right)^{n}$ as we wanted to show.

Remark 4.1. The hypothesis that $\beta<\frac{\gamma(n-m)}{p^{\prime}}-\frac{\gamma-1}{p^{\prime}}$ is necessary in order to have the condition $\int_{\Omega} f=0$ well defined for $f \in L^{p}\left(\Omega, d_{M}^{p \beta}\right)$. Indeed, if $\beta \geq \frac{\gamma(n-m)}{p^{\prime}}-\frac{\gamma-1}{p^{\prime}}$, it is easy to check that $f(x, y, z)=(1-\log x)^{-1} x^{\gamma-1-\gamma(n-m)}$ belongs to $L^{p}\left(\Omega, d_{M}^{p \beta}\right) \backslash$ $L^{1}(\Omega)$.

Remark 4.2. It can be shown that the condition $\eta \geq \beta+\gamma-1$ assumed in the theorem is also necessary. Indeed, if $\eta-\beta<\gamma-1$, it can be shown by generalizations of the example presented in Section 2, that there exists $f \in L_{0}^{p}\left(\Omega, d_{M}^{p \beta}\right)$ such that a solution $\mathbf{u}$ of (4.4) satisfying (4.5) does not exist (see [ADLg] for the details).

\section{Application to the Stokes equations}

In this section we show how the results obtained in the previous section can be applied to prove the well posedness of the Stokes equations in appropriate weighted Sobolev spaces for cuspidal domains. Indeed, combining the variational analysis given in Sections 2 with the results in Section 4 we obtain the following theorem.

Theorem 5.1. Given $\gamma \geq 1$, let $\Omega$ be the domain defined in (4.1). If $\mathbf{g} \in$ $H^{-1}(\Omega)^{n}$ then, there exists a unique $(\mathbf{u}, p) \in H_{0}^{1}(\Omega)^{n} \times L^{2}\left(\Omega, d_{M}^{2(\gamma-1)}\right)$, with $p$ satisfying $\int_{\Omega} p d_{M}^{2(\gamma-1)}=0$, weak solution of the Stokes equations (2.1). Moreover,

$$
\|\mathbf{u}\|_{H_{0}^{1}(\Omega)^{n}}+\|p\|_{L^{2}\left(\Omega, d_{M}^{2(\gamma-1)}\right)} \leq C\|\mathbf{g}\|_{H^{-1}(\Omega)^{n}}
$$

with a constant $C$ depending only on $\gamma$ and $n$.

Proof. Consider the particular case $\eta=0, \beta=1-\gamma$ and $p=2$ in Theorem 4.1. It is easy to check that in this case $\beta$ satisfies the hypothesis of that theorem for any values of $n$ and $m$ (recall that $m \leq n-2$ ), i.e.,

$$
\beta=1-\gamma \in\left(\frac{-\gamma(n-m)}{2}-\frac{\gamma-1}{2}, \frac{\gamma(n-m)}{2}-\frac{\gamma-1}{2}\right) .
$$

Then, given $f \in L_{0}^{2}\left(\Omega, d_{M}^{2(1-\gamma)}\right)$ there exists $\mathbf{u} \in H_{0}^{1}(\Omega)^{n}$ satisfying

$$
\operatorname{div} \mathbf{u}=f
$$

and

$$
\|\mathbf{u}\|_{H^{1}(\Omega)^{n}} \leq C\|f\|_{L^{2}\left(\Omega, d_{M}^{2(1-\gamma)}\right)}
$$

with a constant $C$ depending only on $\gamma$ and $n$. Therefore, the result follows immediately from Theorem 2.1 .

In the next corollary we show the well posedness of the Stokes equations in standard spaces. 
Corollary 5.1. Given $\gamma \geq 1$, let $\Omega$ be the domain defined in (4.1) and $\mathbf{g} \in$ $H^{-1}(\Omega)^{n}$. If $r_{0}$ is defined by

$$
r_{0}=2-\frac{4(\gamma-1)}{\gamma(k+2)-1}
$$

then, $r_{0}>0$, and for $0<r<r_{0}$, there exists a unique $(\mathbf{u}, p) \in H_{0}^{1}(\Omega)^{n} \times L^{r}(\Omega)$, with $p$ satisfying $\int_{\Omega} p d_{M}^{2(\gamma-1)}=0$, weak solution of the Stokes equations (2.1). Moreover, there exists a constant $C$ depending only on $n, \gamma$ and $r$ such that

$$
\|\mathbf{u}\|_{H_{0}^{1}(\Omega)^{n}}+\|p\|_{L^{r}(\Omega)} \leq C\|\mathbf{g}\|_{H^{-1}(\Omega)^{n}}
$$

In particular, if $k \geq 2$, or $k=1$ and $\gamma<3, p \in L^{1}(\Omega)$.

Proof. Since $\gamma>1$ and $k \geq 1$ it follows that $r_{0}>0$. Now, given a positive $r<r_{0}$ it is enough to see that, if $(\mathbf{u}, p)$ is the solution given by Theorem 5.1, then $p \in L^{r}(\Omega)$ and

$$
\|p\|_{L^{r}(\Omega)} \leq C\|p\|_{L^{2}\left(\Omega, d_{M}^{2(\gamma-1)}\right)} .
$$

It is easy to see that $\int_{\Omega} d_{M}^{s}<+\infty$ for any $s>-\gamma k-1$. Then, applying the Hölder inequality with $2 / r$ and its dual exponent we have

$$
\|p\|_{L^{r}(\Omega)}^{r}=\int_{\Omega}|p|^{r} d_{M}^{(\gamma-1) r} d_{M}^{(1-\gamma) r} \leq\|p\|_{L^{2}\left(\Omega, d_{M}^{2(\gamma-1)}\right)}^{r}\left(\int_{\Omega} d_{M}^{\frac{2(1-\gamma) r}{2-r}}\right)^{\frac{2-r}{2}} .
$$

Since $r<r_{0}$, we have $(2(1-\gamma) r) /(2-r)>-\gamma k-1$, and so the integral on the right hand side is finite. Therefore, (5.1) is proved. Finally, if $k \geq 2$, or $k=1$ and $\gamma<3$, it is easy to check that $r_{0}>1$ and therefore $p \in L^{1}(\Omega)$.

To end this section let us show the results of the above theorem and corollary in the particular cases $n=2$ and $n=3$. We will use here the usual notation $x=\left(x_{1}, x_{2}\right) \in \mathbf{R}^{2}$ or $x=\left(x_{1}, x_{2}, x_{3}\right) \in \mathbf{R}^{3}$.

For $n=2$ we have $m=0$ and, for $\gamma \geq 1$, the domain is

$$
\Omega=\left\{x=\left(x_{1}, x_{2}\right) \in \mathbf{R}^{2}: 0<x_{1}<1,\left|x_{2}\right|<x_{1}^{\gamma}\right\} .
$$

In this case $M=(0,0)$ and therefore $d_{M}(x)=|x|$. Then, for $\mathbf{g} \in H^{-1}(\Omega)^{2}$, there exists a unique

$$
(\mathbf{u}, p) \in H_{0}^{1}(\Omega)^{2} \times L^{2}\left(\Omega,|x|^{2(\gamma-1)}\right),
$$

with $p$ satisfying $\int_{\Omega} p|x|^{2(\gamma-1)}=0$, weak solution of the Stokes equations. Moreover,

$$
\|\mathbf{u}\|_{H_{0}^{1}(\Omega)^{2}}+\|p\|_{L^{2}\left(\Omega,|x|^{2(\gamma-1)}\right)} \leq C\|\mathbf{g}\|_{H^{-1}(\Omega)^{2}}
$$

and, for $r<2-\frac{4(\gamma-1)}{3 \gamma-1}$,

$$
\|p\|_{L^{r}(\Omega)} \leq C\|\mathbf{g}\|_{H^{-1}(\Omega)^{2}}
$$

with a constant $C$ depending only on $\gamma$ and $r$.

For $n=3$ we have the two possible cases $m=0$ or $m=1$. In the first case the domain has a cuspidal point and is given by

$$
\Omega=\left\{x=\left(x_{1}, x_{2}, x_{3}\right) \in \mathbf{R}^{3}: 0<x_{1}<1, \sqrt{x_{2}^{2}+x_{3}^{2}}<x_{1}^{\gamma}\right\} .
$$

In this case we obtain exactly the same estimates (5.2) and (5.3) with obvious changes of dimension. The only difference is that now $r<2-\frac{4(\gamma-1)}{4 \gamma-1}$. Observe that in particular, in this case $p \in L^{1}(\Omega)$. 
Finally, when $m=1$, the domain has a cuspidal edge and is given by

$$
\Omega=\left\{x=\left(x_{1}, x_{2}, x_{3}\right) \in \mathbf{R}^{3}: 0<x_{1}<1,0<x_{3}<1,\left|x_{2}\right|<x_{1}^{\gamma}\right\}
$$

and, defining $\bar{x}=\left(x_{1}, x_{2}\right)$, we have $d_{M}(x)=|\bar{x}|$ and the a priori estimates

$$
\|\mathbf{u}\|_{H_{0}^{1}(\Omega)^{3}}+\|p\|_{L^{2}\left(\Omega,|\bar{x}|^{2(\gamma-1)}\right)} \leq C\|\mathbf{g}\|_{H^{-1}(\Omega)^{3}}
$$

and, for $r<2-\frac{4(\gamma-1)}{3 \gamma-1}$,

$$
\|p\|_{L^{r}(\Omega)} \leq C\|\mathbf{g}\|_{H^{-1}(\Omega)^{3}} .
$$

\section{Weighted Korn type inequalities}

Important and well-known consequences of the existence of a right inverse of the divergence operator in Sobolev spaces are the different cases of Korn inequalities. It is also known that the classic first and second cases (in the terminology introduced by Korn) can be derived from the following inequality,

$$
\|D \mathbf{v}\|_{L^{p}(\Omega)^{n \times n}} \leq C\left\{\|\mathbf{v}\|_{L^{p}(\Omega)^{n}}+\|\varepsilon(\mathbf{v})\|_{L^{p}(\Omega)^{n \times n}}\right\},
$$

where we are using the usual notation for the symmetric part of the differential matrix $D \mathbf{v}$ of a vector field $\left(v_{1}, \ldots, v_{n}\right)$, namely,

$$
\varepsilon_{i j}(\mathbf{v})=\frac{1}{2}\left(\frac{\partial v_{i}}{\partial x_{j}}+\frac{\partial v_{j}}{\partial x_{i}}\right) .
$$

For the cuspidal domains that we are considering this inequality is not valid (counterexamples are given in [ADLg, GG, W]). In view of our results on solutions of the divergence it is natural to look for Korn type inequalities in weighted Sobolev spaces. For general Hölder- $\alpha$ domains, inequalities of this kind were obtained in [ADL] using weights which are powers of the distance to the boundary. Here we are interested in stronger results for the particular class of Hölder- $\alpha$ domains defined in (4.1). We are going to prove estimates in norms involving the distance to the cusp.

It is not straightforward to generalize the classic arguments to derive Korn inequalities from the existence of right inverses of the divergence to the weighted case. We do not know how to do it if we work with weighted norms in both sides of the inequality (6.1). Therefore, we are going to prove a result for a general weight and afterwards, we will obtain more general inequalities for the case of weights which are powers of the distance to the cusp, using an argument introduced in [BK].

Let us mention that in what follows we state and prove several inequalities assuming that the left hand side is finite. Afterwards, by density arguments, one can conclude that these inequalities are valid whenever the right hand side is finite. This is a usual procedure. Given $1<p<\infty$, a domain $U \subset \mathbf{R}^{n}$, and a weight $\omega$, we denote with $W^{-1, p^{\prime}}\left(U, \omega^{1-p^{\prime}}\right)$ the dual space of $W_{0}^{1, p}(U, \omega)$. Observe that $W^{-1, p}(U, \omega)=W_{0}^{1, p^{\prime}}\left(U, \omega^{1-p^{\prime}}\right)^{\prime}$.

Lemma 6.1. Given a weight $\omega$, a bounded domain $U \subset \mathbf{R}^{n}$, and $1<p<\infty$, assume that for any $g \in L_{0}^{p^{\prime}}(U)$ there exists $\mathbf{u} \in W_{0}^{1, p^{\prime}}\left(U, \omega^{1-p^{\prime}}\right)^{n}$ such that $\operatorname{div} \mathbf{u}=g$ and

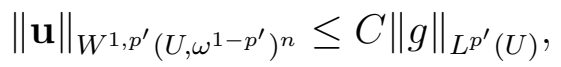

with a constant $C$ depending only on $U, p$, and $\omega$. Fix an open ball $B \subset U$. Then, for any $f \in L^{p}(U)$,

$$
\|f\|_{L^{p}(U)} \leq C\left\{\|f\|_{W^{-1, p}(B)}+\|\nabla f\|_{W^{-1, p}(U, \omega)^{n}}\right\}
$$


where the constant $C$ depends only on $U, B, p$, and $\omega$.

Proof. Take $f \in L^{p}(U)$. If $\bar{f}$ denotes the mean value of $f$ over $U$ we have, for $g \in L^{p^{\prime}}(U)$,

$$
\int_{\mathrm{U}}(f-\bar{f}) g=\int_{\mathrm{U}}(f-\bar{f})(g-\bar{g}) .
$$

But, from our hypothesis, there exists a solution $\mathbf{u} \in W_{0}^{1, p^{\prime}}\left(U, \omega^{1-p^{\prime}}\right)^{n}$ of $\operatorname{div} \mathbf{u}=g-\bar{g}$ satisfying

$$
\|\mathbf{u}\|_{W^{1, p^{\prime}}\left(U, \omega^{1-p^{\prime}}\right)^{n}} \leq C\|g-\bar{g}\|_{L^{p^{\prime}(U)}} .
$$

Thus,

$$
\begin{aligned}
\int_{U}(f-\bar{f}) g=\int_{U}(f-\bar{f}) \operatorname{div} \mathbf{u} & \leq\|\nabla f\|_{W^{-1, p}(U, \omega)^{n}}\|\mathbf{u}\|_{W^{1, p^{\prime}}\left(U, \omega^{1-p^{\prime}}\right)^{n}} \\
& \leq C\|\nabla f\|_{W^{-1, p}(U, \omega)^{n}}\|g-\bar{g}\|_{L^{p^{\prime}}(U)} .
\end{aligned}
$$

Therefore, by duality,

$$
\|f-\bar{f}\|_{L^{p}(U)} \leq C\|\nabla f\|_{W^{-1, p}(U, \omega)^{n}} .
$$

Now, we decompose $f$ as

$$
f=\left(f-f_{\varphi}\right)+f_{\varphi}
$$

where $f_{\varphi}:=\int_{B} f \varphi$ with $\varphi \in C_{0}^{\infty}(B)$ such that $\int_{B} \varphi=1$. Thus,

$$
f-f_{\varphi}=f-\bar{f}+\int_{B}(\bar{f}-f) \varphi
$$

and so, using (6.2),

$$
\left\|f-f_{\varphi}\right\|_{L^{p}(U)} \leq\left(1+\|\varphi\|_{L^{p^{\prime}}(B)}\right)\|f-\bar{f}\|_{L^{p}(U)} \leq C\|\nabla f\|_{W^{-1, p}(U, \omega)^{n}} .
$$

Therefore, to conclude the proof we have to estimate $\left\|f_{\varphi}\right\|_{L^{p}(U)}$. But,

$$
\left\|f_{\varphi}\right\|_{L^{p}(U)} \leq|U|^{1 / p}\left|\int_{B} f \varphi\right| \leq|U|^{1 / p}\|f\|_{W^{-1, p}(B)}\|\varphi\|_{W_{0}^{1, p^{\prime}(B)}} .
$$

Using this lemma we can generalize a classic argument to prove a Korn type inequality obtaining the following result.

Theorem 6.1. Given a weight $\omega$, a bounded domain $U \subset \mathbf{R}^{n}$, and $1<p<\infty$, assume that for any $g \in L_{0}^{p^{\prime}}(U)$ there exists $\mathbf{u} \in W_{0}^{1, p^{\prime}}\left(U, \omega^{1-p^{\prime}}\right)^{n}$ such that $\operatorname{div} \mathbf{u}=g$ and

$$
\|\mathbf{u}\|_{W^{1, p^{\prime}}\left(U, \omega^{1-p^{\prime}}\right)^{n}} \leq C\|g\|_{L^{p^{\prime}(U)}},
$$

with a constant $C$ depending only on $U, p$, and $\omega$. Fix an open ball $B \subset U$. Then, for any $\mathbf{v} \in W^{1, p}(U)^{n}$,

$$
\|D \mathbf{v}\|_{L^{p}(U)^{n \times n}} \leq C\left\{\|\mathbf{v}\|_{L^{p}(B)^{n}}+\|\varepsilon(\mathbf{v})\|_{L^{p}(U, \omega)^{n \times n}}\right\},
$$

where the constant $C$ depends only on $U, B, p$, and $\omega$.

Proof. It is known that, for any $g \in L^{p}(B)$,

$$
\left\|\frac{\partial g}{\partial x_{j}}\right\|_{W^{-1, p}(B)} \leq\|g\|_{L^{p}(B)} .
$$


Analogously, for any $g \in L^{p}(U, \omega)$, we have

$$
\left\|\frac{\partial g}{\partial x_{j}}\right\|_{W^{-1, p}(U, \omega)}=\sup _{0 \neq \phi \in W_{0}^{1, p^{\prime}}\left(U, \omega^{1-p^{\prime}}\right)} \frac{\left|\int_{U} g \frac{\partial \phi}{\partial x_{j}}\right|}{\|\phi\|_{W^{1, p^{\prime}}\left(U, \omega^{1-p^{\prime}}\right)}} \leq\|g\|_{L^{p}(U, \omega)} .
$$

On the other hand, applying Lemma 6.1, we have

$$
\left\|\frac{\partial v_{i}}{\partial x_{j}}\right\|_{L^{p}(U)} \leq C\left\{\left\|\frac{\partial v_{i}}{\partial x_{j}}\right\|_{W^{-1, p}(B)}+\left\|\nabla \frac{\partial v_{i}}{\partial x_{j}}\right\|_{W^{-1, p}(U, \omega)^{n}}\right\} .
$$

Using now the well known identity

$$
\frac{\partial^{2} v_{i}}{\partial x_{j} \partial x_{k}}=\frac{\partial \varepsilon_{i k}(\mathbf{v})}{\partial x_{j}}+\frac{\partial \varepsilon_{i j}(\mathbf{v})}{\partial x_{k}}-\frac{\partial \varepsilon_{j k}(\mathbf{v})}{\partial x_{i}}
$$

in the last term on the right hand side, and the inequalities (6.3) and (6.4), we conclude the proof.

An immediate consequence of Theorems 6.1 and 4.1 is the following.

Corollary 6.1. Given $\gamma \geq 1$, let $\Omega$ be the domain defined in (4.1), $M$ defined in (4.2), $1<p<\infty$, and $B \subset \Omega$ an open ball. Then, there exists a constant $C$, which depends only on $\Omega, B$, and $p$, such that for all $\mathbf{u} \in W^{1, p}(\Omega)^{n}$,

$$
\|D \mathbf{u}\|_{L^{p}(\Omega)^{n \times n}} \leq C\left\{\|\mathbf{u}\|_{L^{p}(B)^{n}}+\|\varepsilon(\mathbf{u})\|_{L^{p}\left(\Omega, d_{M}^{p(1-\gamma)}\right)^{n \times n}}\right\} .
$$

Proof. According to Theorem 4.1, for any $g \in L_{0}^{p^{\prime}}(\Omega)$ there exists $\mathbf{u} \in W_{0}^{1, p^{\prime}}(\Omega$, $\left.d_{M}^{p^{\prime}(\gamma-1)}\right)^{n}$ such that $\operatorname{div} \mathbf{u}=g$ and

$$
\|\mathbf{u}\|_{W^{1, p^{\prime}}\left(\Omega, d_{M}^{p^{\prime}(\gamma-1)}\right)^{n}} \leq C\|g\|_{L^{p^{\prime}}(\Omega)},
$$

with a constant $C$ depending only on $\gamma$ and $p$. Therefore, Theorem 6.1 applies for $\omega=d_{M}^{p(1-\gamma)}$.

We conclude the paper proving more general Korn type inequalities for the cuspidal domains defined in (4.1). To obtain these inequalities we use an argument introduced in [BK].

Theorem 6.2. Given $\gamma \geq 1$, let $\Omega$ be the domain defined in (4.1), $M$ defined in (4.2), $1<p<\infty, B \subset \Omega$ an open ball, and $\beta \geq 0$. Then, there exists a constant $C$, which depends only on $\Omega, B, p$, and $\beta$, such that for all $\mathbf{u} \in W^{1, p}\left(\Omega, d_{M}^{p \beta}\right)^{n}$

$$
\|D \mathbf{u}\|_{L^{p}\left(\Omega, d_{M}^{p \beta}\right)^{n \times n}} \leq C\left\{\|\mathbf{u}\|_{L^{p}(B)^{n}}+\|\varepsilon(\mathbf{u})\|_{L^{p}\left(\Omega, d_{M}^{p(\beta+1-\gamma)}\right)^{n \times n}}\right\} .
$$

Proof. To simplify the notation we will assume that $m=0$ in the definition of $\Omega$. The other cases can be treated analogously.

Let $n^{\prime} \in \mathbf{N}_{0}$ and $0<s \leq \gamma$ be such that $s n^{\prime}=p \beta$. As in $[\mathrm{BK}]$ we introduce

$$
\Omega^{n^{\prime}, s}=\left\{\left(x, y, z^{\prime}\right) \in \mathbf{R}^{n+n^{\prime}}:(x, y) \in \Omega, z^{\prime} \in \mathbf{R}^{n^{\prime}} \text { with }\left|z^{\prime}\right|<x^{s}\right\} .
$$

Suppose that the hypothesis in Theorem 6.1 on solutions of the divergence is verified for $U=\Omega^{n^{\prime}, s}$ and $\omega=x^{p(1-\gamma)}$. Then, if $B^{\prime} \subset \Omega^{n^{\prime}, s}$ is a ball with the same radius and center than $B$, we have

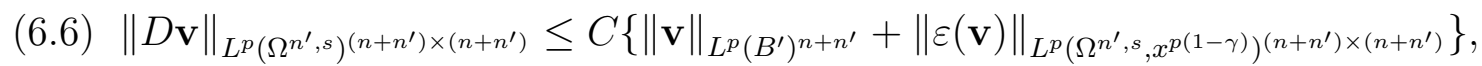
for all $\mathbf{v} \in W^{1, p}\left(\Omega^{n^{\prime}, s}\right)^{n+n^{\prime}}$. 
Now, given $\mathbf{u}$ in $W^{1, p}\left(\Omega, d_{M}^{p \beta}\right)^{n}$ we define

$$
\mathbf{v}\left(x, y, z^{\prime}\right)=(\mathbf{u}(x, y), \underbrace{0, \ldots, 0}_{n^{\prime}}) .
$$

Then, using that for $(x, y) \in \Omega, d_{M}(x, y) \simeq x$, it is easy to check that (6.6) is equivalent to

$$
\|D \mathbf{u}\|_{L^{p}\left(\Omega, d_{M}^{p \beta}\right)^{n \times n}} \leq C\left\{\|\mathbf{u}\|_{L^{p}(B)^{n}}+\|\varepsilon(\mathbf{u})\|_{L^{p}\left(\Omega, d_{M}^{p(\beta+1-\gamma)}\right)^{n \times n}}\right\} .
$$

Hence, to finish the proof we have to verify the hypothesis of Theorem 6.1 for the domain $\Omega^{n^{\prime}, s}$ with the weight $\omega=x^{p(1-\gamma)}$. Since in this case $\omega^{1-p^{\prime}}=x^{p^{\prime}(\gamma-1)}$, we have to show that, for any $g \in L_{0}^{p^{\prime}}\left(\Omega^{n^{\prime}, s}\right)$, there exists $\mathbf{w} \in W_{0}^{1, p^{\prime}}\left(\Omega^{n^{\prime}, s}, x^{p^{\prime}(\gamma-1)}\right)^{n}$ such that $\operatorname{div} \mathbf{w}=g$ and

$$
\|\mathbf{w}\|_{W^{1, p^{\prime}}\left(\Omega^{n^{\prime}, s}, x^{p^{\prime}(\gamma-1)}\right)^{n+n^{\prime}}} \leq C\|g\|_{L^{p^{\prime}\left(\Omega^{n^{\prime}, s}\right)}} .
$$

But this can be proved exactly as Theorem 4.1, using now the convex domain

$$
\widehat{\Omega}^{n^{\prime}, s}:=\left\{\left(\hat{x}, \hat{y}, \hat{z}^{\prime}\right) \in \mathbf{R}^{n+n^{\prime}}:(\hat{x}, \hat{y}) \in \widehat{\Omega}, z^{\prime} \in \mathbf{R}^{n^{\prime}} \text { with }\left|z^{\prime}\right|<x^{\alpha s}\right\},
$$

with $\widehat{\Omega}$ defined as in (4.6), and the one-to-one map $F: \widehat{\Omega}^{n^{\prime}, s} \rightarrow \Omega^{n^{\prime}, s}$ defined by

$$
F\left(\hat{x}, \hat{y}, \hat{z}^{\prime}\right):=\left(\hat{x}^{\alpha}, \hat{y}, \hat{z}^{\prime}\right) \text {. }
$$

\section{References}

[ADL] Acosta, G., R. G. Durán, and A. L. Lombard: Weighted Poincaré and Korn inequalities for Hölder $\alpha$ domains. - Math. Methods Appl. Sci. 29:4, 2006, 387-400.

[ADLg] Acosta, G., R. G. Durán, and F. López García: Work in progress.

[ADM] Acosta, G., R. G. DurÁn, and M. A. Muschietti: Solutions of the divergence operator on John domains. - Adv. Math. 206:2, 2006, 373-401.

[ASV] ARnold, D. N., L. R. Scott, and M. Vogelius: Regular inversion of the divergence operator with Dirichlet boundary conditions on a polygon. - Ann. Sc. Norm. Super. Pisa Cl. Sci. (4) 15, 1988, 169-192.

[BA] Babuska, I., and A. K. AzIZ: Survey lectures on the mathematical foundation of the finite element method. - In: The mathematical foundations of the finite element method with applications to partial differential equations, edited by A.K. Aziz, Academic Press, New York, 1972, 5-539.

[BDF] Boffi, D., F. Brezzi, L. F. Demkowicz, R. G. Durán, R. S. Falk, and M. Fortin: Finite elements, compatibility conditions, and applications. - Lecture Notes in Math. 1939, Springer-Verlag, Berlin.

[B] Bogovskit, M. E., Solution of the first boundary value problem for the equation of continuity of an incompressible medium. - Soviet Math. Dokl. 20, 1979, 1094-1098.

[BS] Brenner, S. C., and L. R. Scott: The mathematical theory of finite element methods. Springer-Verlag, Berlin, 1994.

[Br] BrezzI, F.: On the existence, uniqueness and approximation of saddle-point problems arising from Lagrangian multipliers. - Rev. Française Automat. Informat. Recherche Opérationnelle Sér. Rouge 8, 1974, 129-151.

[BF] Brezzi, F., and M. Fortin: Mixed and hybrid finite element methods. - Springer Ser. Comput. Math. 15, Springer-Verlag, New York, 1991.

[BK] Buckley, S. M., and P. Koskela: New Poincaré inequalities from old. - Ann. Acad. Sci. Fenn. Math. 23, 1998, 251-260. 
[CZ] Calderón, A. P., and A. Zygmund: On singular integrals. - Amer. J. Math. 78, 1956, 289-309.

[C] Ciarlet, P. G.: Introduction to linear shell theory. - Ser. Appl. Math., P. G. Ciarlet and P. L. Lions, eds., Gauthier-Villars, 1998.

[DRS] Diening, L., M. RŮŽIČKa, and K. Schumacher: A decomposition technique for John domains. - Ann. Acad. Sci. Fenn. Math. 35, 2010, 87-114.

[Du] Duonndikoetxea, J.: Fourier analysis. - Grad. Stud. Math. 29, Amer. Math. Soc., 2001.

[D] DurÁn, R. G.: The inf-sup condition for the Stokes equations: A constructive approach in general domains. - Mathematisches Forschungsinstitut Oberwolfach, Workshop on Gemischte und nicht-standard Finite-Elemente-Methoden mit Anwendungen, Extended abstract, Report No. 5, 2005, 270-272.

[DLg] Durán, R. G., and F. López García: Solutions of the divergence and analysis of the Stokes equations in planar Hölder- $\alpha$ domains. - Math. Models Methods Appl. Sci. 20:1, 2010, 95-120.

[DM] DurÁn, R. G., and M. A. Muschietti: An explicit right inverse of the divergence operator which is continuous in weighted norms. - Studia Math. 148:3, 2001, 207-219.

[F] FriedRICHS, K. O.: On the boundary-value problems of the theory of elasticity and Korn's inequality. - Ann. of Math. (2) 48, 1947, 441-471.

[G] Galdi, G. P.: An introduction to the mathematical theory of the Navier-Stokes equations. Vol. I. Linearized steady problems. - Springer Tracts Nat. Philos. 38, Springer-Verlag, New York, 1994.

[GG] Geymonat, G., and G. Gilard: Contre-exemples á l'inégalité de Korn et au Lemme de Lions dans des domaines irréguliers. - Équations aux Dérivées Partielles et Applications, Gauthiers-Villars, Éd. Sci. Méd. Elsevier, Paris, 1998, 541-548.

[GR] Girault, V., and P. A. Raviart: Finite element methods for Navier-Stokes equations. Springer-Verlag, Berlin, 1986.

[GU] Gol'Dshtein, V., and A. Ukhlov: Weighted Sobolev spaces and embedding theorems. Trans. Amer. Math. Soc. 361, 2009, 3829-3850.

[Ki] Kilpeläinen, T.: Weighted Sobolev spaces and capacity. - Ann. Acad. Sci. Fenn. Ser. A I Math. 19, 1994, 95-113.

[Ku] Kufner, A.: Weighted Sobolev spaces. - John Wiley \& Sons, New York, 1985.

[L] Ladyzhenskaya, O. A.: The mathematical theory of viscous incompressible flow. - Gordon and Breach, New York, 1969.

[N] Nicolás, F.: Inverse of the divergence in Hardy spaces on star-shaped domains. - Preprint, 2008.

[S1] Stein, E. M.: Note on singular integrals. - Proc. Amer. Math. Soc. 8:2, 1957, 250-254.

[S2] Stein, E. M.: Harmonic analysis: real-variable methods, orthogonality, and oscillatory integrals. - Princeton Math. Ser. 43, Monographs in Harmonic Analysis III, Princeton Univ. Press, Princeton, NY, 1993.

[W] WECK, N.: Local compactness for linear elasticity in irregular domains. - Math. Meth. Appl. Sci. 17, 1994, 107-113. 